\title{
Attitude-Independent Magnetometer Calibration with Time-Varying Bias
}

\author{
John C. Springmann \\ University of Michigan, Ann Arbor \\ Adviser: James W. Cutler
}

\begin{abstract}
We present a method for on-orbit, attitude-independent magnetometer calibration that includes the effect of time-varying bias due to electronics on-board a spacecraft. The calibration estimates magnetometer scale factors, mis-alignments, and constant as well as time-varying bias. Time-varying effects are mitigated by including spacecraft telemetry in the measurement model and estimating constant parameters that map the telemetry data to magnetometer bias. The calibration is demonstrated by application to flight data from the Radio Aurora Explorer satellite and significantly reduces the uncertainty of off-the-shelf magnetometers embedded within the satellite and subject to spacecraftgenerated fields. This method simplifies the satellite design process by reducing the need for booms and strict magnetic cleanliness requirements.
\end{abstract}

\section{Introduction}

This paper presents a method for attitudeindependent, on-orbit magnetometer calibration that mitigates the effect of time-varying magnetic fields produced by electronics on-board a spacecraft. The calibration significantly increases the accuracy of measurements from magnetometers embedded within a satellite. We are motivated by improved magnetometerbased attitude determination, but this calibration is applicable to magnetometers in general. Three-axis magnetometers are a common sensor on low-Earth orbiting spacecraft because they are reliable, lightweight, have low power requirements, and have no moving parts [4]. Their applications include attitude determination and scientific measurements.

In general, three-axis magnetometer measurements are corrupted by hard iron errors, soft iron errors, scale factors, and non-orthogonality errors [9]. Hard iron error is a magnetic field bias that is either constant, which is caused by ferromagnetic (hard iron) materials near the magnetometer, or time-varying, which is caused by current carrying wires near the magnetometer. Soft iron errors result from materials that generate fields in response to externally applied fields (commonly called soft iron materials), resulting in scaling of magnetometer readings. Scale factor errors are inherent to the sensor and result from different sensitivities in each axis of the sensor. Nonorthogonality errors are due to angular deviation from a perfectly orthogonal three-axis configuration, and can be caused by manufacturing errors, thermal stress, or mechanical stress.

Existing calibration methods group all the sources of error into three parameters: bias, scaling factors, and misalignments. For example, TWOSTEP is an attitudeindependent algorithm that estimates magnetometer bias $[1,3]$ and has been extended to estimate scale factors and non-orthogonality corrections [2]. Another calibration technique to estimate bias, scale factors, and nonorthogonality is developed in References 8,9,14. These algorithms $[1-3,8,9,14]$ are batch methods, meaning they are post-processing techniques applied using many measurements simultaneously. A real-time implementation of Reference 2 is developed by Crassidis et al [5]. The algorithms are also attitude-independent, meaning no attitude knowledge is required for the calibration. This is critical because the magnetometers are used to estimate attitude, so attitude is generally not available before calibration.

The existing algorithms assume the scale factors, biases, and misalignments are constant. In reality, there is an additional time-varying bias caused by electronic components. Traditionally, this bias is minimized by either using a boom to extend the magnetometer away from the sources of bias, or by using costly design and manufacturing practices to minimize the influence of electronic components on magnetometers.

In this paper, we expand the work of Foster and Elkaim [8] to take time-varying bias into account. The calibration is particularly useful for small satellites, for which volume constraints can prevent sufficient physical 
separation between magnetometers and sources of timevarying bias, and cost and time constraints are not conducive to designing a boom or a magnetically clean satellite bus. The use of the calibration technique reduces the need for booms and magnetic cleanliness requirements, which results in potentially lower satellite development times and costs.

The remainder of this paper is organized as follows. In Section II, we review the techniques of Foster and Elkaim [8] and apply the method to flight data from the Radio Aurora Explorer (RAX) [7] satellite. The results motivate modifications to the calibration technique to include time-varying bias, which are discussed in Section III. Application to flight data is presented in Section IV and conclusions are discussed in Section V.

\section{Application of Existing Calibration Method to Flight Data}

The calibration algorithm presented in this paper is an extension of the technique developed in References 8 and 9. For completeness, we present the original algorithm here, and then present the modifications developed for improved on-orbit calibration in Section III.

\section{A. Attitude-Independent Calibration Technique}

The key to this method $[8,9]$ is the fact that the plot of the output of an ideal three-axis magnetometer lies on a sphere with radius equal to the magnitude of the true magnetic field. That is, when a perfect three-axis magnetometer is rotated arbitrarily in a constant magnetic field, a 3D plot of the components of the magnetic field will form a sphere. This can be seen from the equation

$$
B^{2}=B_{x}^{2}+B_{y}^{2}+B_{z}^{2},
$$

where $B_{x}, B_{y}$, and $B_{z}$ are components of the magnetic field in the sensor frame, and $B$ is the magnitude of the magnetic field. This matches the form of the equation of a sphere centered at $(0,0,0)$ with components $B_{x}, B_{y}$, and $B_{z}$ and a radius of $B$. Sensor bias will cause the origin of the sphere to be offset from $(0,0,0)$, and axis nonorthogonality and scaling will transform the sphere into an ellipsoid. This can be shown mathematically $[8,9,14]$, but the derivation is omitted here.

In addition to Eq. (1), a model of each axis of the magnetometer is used. The model includes a scaling factor, total offset, and axis non-orthogonality. We start by including the scaling factor and offset in each axis. The scaling factor represents both scaling inherent to the sensor and soft iron effects; it is not possible to mathematically separate each physical contribution [8]. The offsets include both constant bias and any null shift inherent to the sensor. The model is given by Eqs. (2)-(4), where $\tilde{B}_{x}$, $\tilde{B}_{y}$, and $\tilde{B}_{z}$ are the measured magnetic field components, $B_{x}, B_{y}$, and $B_{z}$ are the true magnetic field components, $a, b$, and $c$ are the scaling factors of each axis, and $x_{0}, y_{0}$, and $z_{0}$ are the offsets. Measurement noise is not explicitly included in the model, so the offset terms will include a contribution from sensor noise.

$$
\begin{gathered}
\tilde{B}_{x}=a B_{x}+x_{0} \\
\tilde{B}_{y}=b B_{y}+y_{0} \\
\tilde{B}_{z}=c B_{z}+z_{0}
\end{gathered}
$$

Next, axis non-orthogonality is added to the model. The calibration is performed in the sensor frame, so misalignments are given relative to the actual sensor axes. The sensor $x$-axis is chosen as the reference axis, and the misalignments are described using the convention shown in Figure 1, where $\rho$ is the angle from the nominal $y$-axis in the $x$ - $y$ plane, and $\phi$ and $\lambda$ are the angles from the nominal $z$-axis in the $y-z$ and $x-z$ planes, respectively. This results in Eqs. (5)-(7).

$$
\begin{gathered}
\tilde{B}_{x}=a B_{x}+x_{0} \\
\tilde{B}_{y}=b\left(B_{y} \cos (\rho)+B_{x} \sin (\rho)\right)+y_{0}
\end{gathered}
$$

$$
\begin{gathered}
\tilde{B}_{z}=c\left(B_{x} \sin (\lambda)+B_{y} \sin (\phi) \cos (\lambda)+\right. \\
\left.B_{z} \cos (\phi) \cos (\lambda)\right)+z_{0}
\end{gathered}
$$

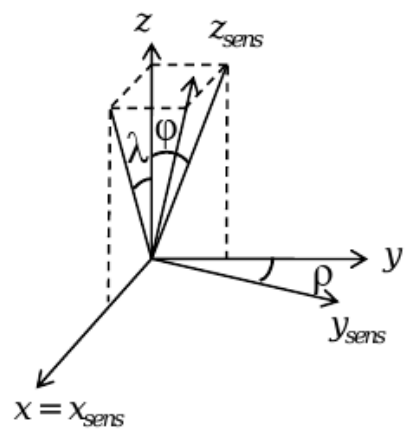

Figure 1. Convention for the angular mis-alignments, where $x, y$, and $z$ are perfectly orthogonal axes, and $x_{\text {sens }}, y_{\text {sens }}$, and $z_{\text {sens }}$ are the sensor axes.

Solving for $B_{x}, B_{y}$, and $B_{z}$ in Eqs. (5)-(7) followed by substitution into Eq. (1) yields a non-linear equation. To estimate the calibration parameters, intermediate variables are used to form the linear equation

$$
\begin{array}{r}
A \tilde{B}_{x}^{2}+B \tilde{B}_{x} \tilde{B}_{y}+C \tilde{B}_{x} \tilde{B}_{z}+D \tilde{B}_{y}^{2}+E \tilde{B}_{y} \tilde{B}_{z}+ \\
F \tilde{B}_{z}^{2}+G \tilde{B}_{x}+H \tilde{B}_{y}+I \tilde{B}_{z}+J=0
\end{array}
$$


where the intermediate variables $A, B, C, D, E, F, G$, $H, I$, and $J$ are nonlinear functions of the calibration parameters $a, b, c, x_{0}, y_{0}, z_{0}, \rho, \phi$, and $\lambda$. A two-step process is used to estimate the calibration parameters. First, since Eq. (8) is linear, batch linear least-squares is used to estimate the coefficients $A-J$. Assuming a constant field magnitude $B$, the calibration parameters $a-\lambda$ can then be determined algebraically from $A-J$. The estimates of $A-J$ are optimal in the least-squares sense, but the parameters $a-\lambda$ are not necessarily optimal. Even so, this method provides very good estimates [8].

This calibration requires knowledge of the magnitude of the ambient magnetic field. No vector knowledge of the field is required, which makes the calibration independent of attitude. For calibration with on-orbit sensor data, we use the magnitude of the International Geomagnetic Reference Field (IGRF), which is expected to be accurate to $10 \mathrm{nT}$ during normal space weather conditions [11]. The corresponding angular accuracy is dependent on the field strength. For the RAX orbit, the field strength is between $20 \mu \mathrm{T}$ and $50 \mu \mathrm{T}$, so $10 \mathrm{nT}$ field uncertainty corresponds to approximate angular uncertainties between $0.03^{\circ}$ and $0.01^{\circ}$.

\section{B. Numerical Solution}

As described above, after the linear least-squares is used to estimate the coefficients $A$ - $J$, the calibration parameters are determined algebraically. This is only possible if the magnetic field magnitude used for the calibration is constant, which is not the case on-orbit. The first modification we make to the algorithm is to estimate the calibration parameters numerically, which allows for calibration with a dynamic field magnitude.

We use non-linear least-squares to estimate the calibration parameters from the on-orbit measurements. The general measurement model is [6]

$$
\overrightarrow{\tilde{y}}=f(\vec{x})+\vec{\nu}
$$

where $\overrightarrow{\tilde{y}}$ is the $m \times 1$ measurement vector, $\vec{x}$ is the state vector to be estimated, and $\vec{\nu}$ is the $m \times 1$ measurement noise vector. Non-linear least-squares is used to iteratively find an estimate of the state vector, $\hat{\vec{x}}$, that minimizes the loss function

$$
J=\frac{1}{2}[\overrightarrow{\tilde{y}}-f(\hat{\vec{x}})]^{T}[\overrightarrow{\tilde{y}}-f(\hat{\vec{x}})] .
$$

Newton's root solving method is used to minimize Eq. (10), so the Jacobian of $f(\vec{x}), \frac{\partial f(\vec{x})}{\partial \vec{x}}$, is required.

For application to estimation of the magnetometer calibration parameters, Eq. (9) takes the form

$$
\overrightarrow{B^{2}}=f\left(\vec{x}, \overrightarrow{\tilde{B}}_{x}, \overrightarrow{\tilde{B}}_{y}, \overrightarrow{\tilde{B}}_{z}\right)+\vec{\nu}
$$

where $\overrightarrow{B^{2}}$ is the $m \times 1$ vector of magnetic field magnitudes squared, $\overrightarrow{\tilde{B}}_{x}, \overrightarrow{\tilde{B}}_{y}$, and $\overrightarrow{\tilde{B}}_{z}$ are the $m \times 1$ measurement vectors, $\vec{x}=\left[\begin{array}{lllllllll}a & b & c & x_{0} & y_{0} & z_{0} & \rho & \phi & \lambda\end{array}\right]^{T}$ is the state vector to be estimated, and $\vec{\nu}$ is measurement noise.

We used two methods to verify that the numerical algorithm converges to the correct estimates. First, we applied both the two-step and numerical methods to experimental data obtained from pre-flight ground testing in a constant magnetic field, and the two methods produced the same estimates. Additionally, simulated testing was used to verify the numerical algorithm converges even with large initial condition errors.

\section{Application to Flight Data}

We now apply the algorithm to flight data from the RAX satellite [7]. RAX is a $3 \mathrm{U}^{1}$ CubeSat that was launched November 19, 2010 into a $650 \mathrm{~km}, 72^{\circ}$ inclination circular orbit. RAX has two three-axis magnetometers embedded within the satellite, along with four two-axis magnetometers mounted on the solar panels. All magnetometers are commercial off-the-shelf components integrated into a custom attitude determination system. In this work, we will use data from the two three-axis magnetometers, which are a PNI MicroMag3 and an Analog Devices ADIS 16405. We will refer to the magnetometers as PNI and $I M U$, respectively (IMU because the ADIS 16405 is an inertial measurement unit that includes a magnetometer). We show $1 \mathrm{~Hz}$ data taken over approximately one orbit starting December 1, 2010 at 08:30:46 UTC. The $\mathrm{Kp}$ inde $\mathrm{x}^{2}$ during this time was $0+$, which indicates quiet magnetic conditions and validates the assumption that the IGRF will provide accurate data for this time period.

Figure 2 shows the magnitude of raw, uncalibrated data from the PNI magnetometer, along with the magnitude of the data corrected by the calibration. The data is overlaid with the magnitude of IGRF data. RAX has a GPS system on-board, but it was not activated during this time period, so position is determined from a Two Line Element set [13].

We see that although the calibration yields drastic improvements over the raw data, the calibrated measurements still have deviations of up to $6 \mu \mathrm{T}$ ( $25 \%$ of the total measurement). To study the calibration further and look for time-varying effects, we look at the difference between the calibrated PNI and IMU readings. If the calibration is perfect, and the two magnetometers are perfectly aligned, then the measurements would match. The difference is shown for each individual magnetometer axis in Figure 3.

\footnotetext{
${ }^{1} 3 \mathrm{U}$ is the designation for a triple CubeSat, which is approximately $10 \mathrm{~cm} \times 10 \mathrm{~cm} \times 30 \mathrm{~cm}$ and $3 \mathrm{~kg}$.

${ }^{2} \mathrm{Kp}$ index retrieved from the NOAA National Geophysical Data Center, http://www.ngdc.noaa.gov/stp/geomag/kp_ap.html
} 


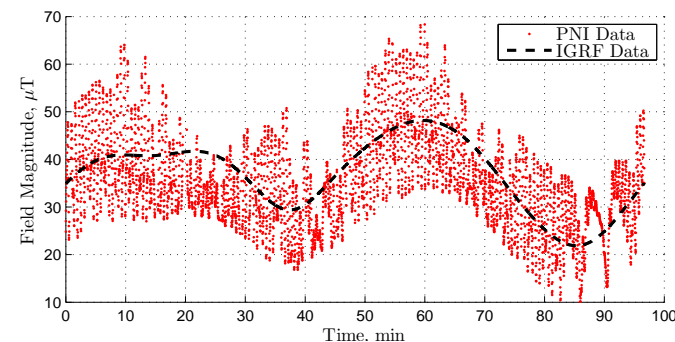

a) Uncalibrated PNI measured field magnitude

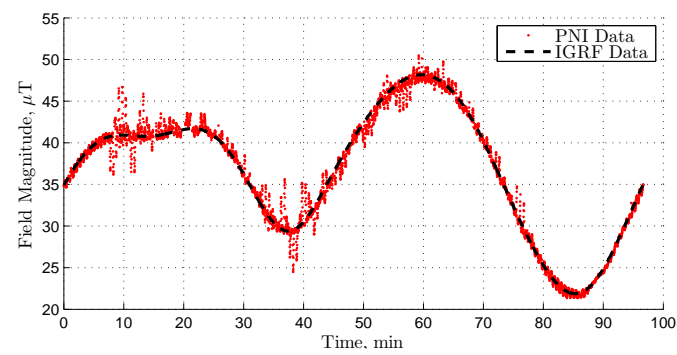

b) PNI measured field magnitude after calibration. The calibration does not take time-varying parameters into account. We see that although the data is improved compared to Figure 2a), there are errors of up to $6 \mu \mathrm{T}$.

Figure 2. Field magnitude versus time for data from the PNI magnetometer overlaid with IGRF data.

The data is overlaid with an indicator that takes the value of one if RAX is in the sun and zero if in eclipse. The indicator is based on sun sensor measurements. We see that the difference in the readings is clearly greater when in the sun, which indicates that currents generated by the solar panels are adversely affecting the magnetometers. This is seen especially in the $x$ and $y$ axes. This data confirms that the magnetometers are affected by time-varying bias due to electronics on-board RAX.

\section{Calibration with Time-Varying Bias}

We take time-varying bias into account by including spacecraft telemetry in the magnetometer model. Sensors on-board monitor current and voltage of various subsystems in the spacecraft. Since current-carrying wires create magnetic fields, we include current measurements in the magnetometer model and estimate constant parameters that map the time-varying current to magnetometer bias.

Because of the dependence of solar power shown in Figure 3, we start by adding the current in each of the solar panels to the model. RAX has four solar panels, one on each of the approximately $10 \times 30 \mathrm{~cm}^{2}$ faces, and the names of the faces are $+x,-x,+y$, and $-y$ (the names correspond to the body-fixed coordinate system).

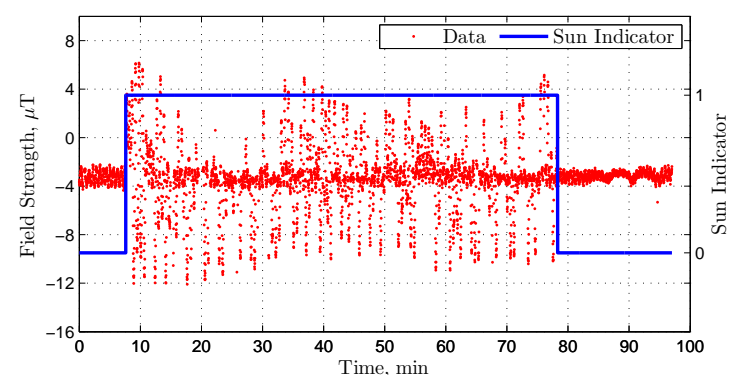

a) $\mathbf{x}$-axis

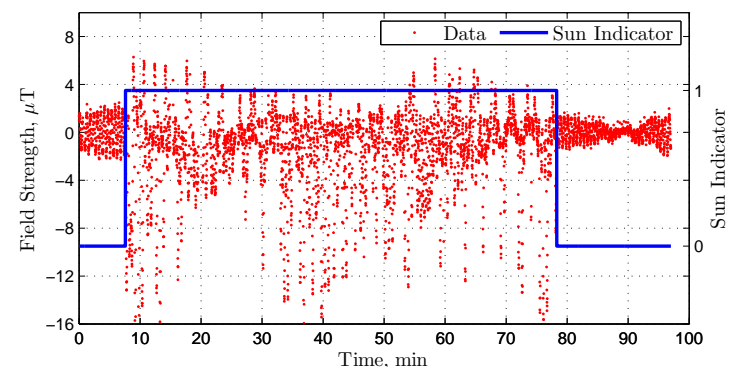

b) $\mathbf{y}$-axis

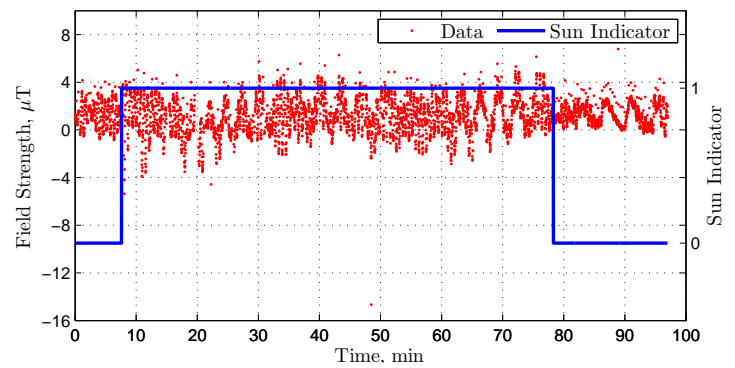

c) z-axis

Figure 3. Difference between IMU and PNI measured components over time after the calibration has been applied. A sun indicator is overlaid that takes the value one when RAX is in the sun and zero when in eclipse. This shows that the solar panel currents are adversely affecting the magnetometers.

We use current telemetry from each face, $\tilde{I}_{+x}, \tilde{I}_{-x}, \tilde{I}_{+y}$, and $\tilde{I}_{-y}$. With the addition of the current measurements to the model given by Eqs. (5)-(7), the model becomes

$$
\begin{aligned}
\tilde{B}_{x}= & a B_{x}+x_{0}+s_{x,+x} \tilde{I}_{+x}+ \\
& s_{x,-x} \tilde{I}_{-x}+s_{x,+y} \tilde{I}_{+y}+s_{x,-y} \tilde{I}_{-y}, \\
\tilde{B}_{y}= & b\left(B_{y} \cos (\rho)+B_{x} \sin (\rho)\right)+y_{0}+ \\
& s_{y,+x} \tilde{I}_{+x}+s_{y,-x} \tilde{I}_{-x}+s_{y,+y} \tilde{I}_{+y} \\
& +s_{y,-y} \tilde{I}_{-y}, \\
\tilde{B}_{z}=\quad & c\left(B_{x} \sin (\lambda)+B_{y} \sin (\phi) \cos (\lambda)+\right. \\
& \left.B_{z} \cos (\phi) \cos (\lambda)\right)+z_{0}+s_{y,+x} \tilde{I}_{+x}+ \\
& s_{y,-x} \tilde{I}_{-x}+s_{y,+y} \tilde{I}_{+y}+s_{y,-y} \tilde{I}_{-y} .
\end{aligned}
$$


The coefficients $s_{i, j}, i \in\{x, y, z\}, j \in\{+x,-x,+y,-y\}$, map the current of the $j$-th panel to the resulting magnetic field bias in the $i$-th magnetometer axis. In general, the magnetic field caused by current-carrying wires is a function of the relative position and orientation of the wires, and the coefficients $s_{i, j}$ are a simple way to take these parameters into account. There is a unique solution to each coefficient only if the current measurements are independent. That is, when stacking $m$ measurements into an $m \times 1$ column, each column of current measurements must be linearly independent. This why it is not possible to mathematically estimate an arbitrarily high number of scaling factor or offset parameters.

With the modified magnetometer model in-hand, we proceed with the same steps described in Section IIB. We solve Eqs. (12)-(14) for $B_{x}, B_{y}$, and $B_{z}$, and substitute into Eq. (1). We use the IGRF magnitude and non-linear least-squares to estimate each calibration parameter. Eq. (11) becomes

$$
\overrightarrow{B^{2}}=f\left(\vec{x}, \overrightarrow{\tilde{B}}_{x}, \overrightarrow{\tilde{B}}_{y}, \overrightarrow{\tilde{B}}_{z}, \overrightarrow{\tilde{I}}_{+x}, \overrightarrow{\tilde{I}}_{-x}, \overrightarrow{\tilde{I}}_{+y}, \overrightarrow{\tilde{I}}_{-y}\right)+\vec{\nu}
$$

and the state vector $\vec{x}$ now contains 21 parameters: the 9 parameters of the original model and 12 coefficients $s_{i, j}$. Simulated testing has been used to verify that the numerical algorithm converges to correct calibration parameters given simulated noisy measurements generated with the model of Eqs. (12)-(14).

In this section, we have presented the model used specifically to include current telemetry from four solar panels. But this method is general so the model can be expanded to include other telemetry points in the same manner. Such expansion could be done to enhance the calibration if additional applicable telemetry points are available.

\section{Application of Time-Varying Calibration to Flight Data}

We now apply the calibration technique to RAX flight data. The results of calibration with the solar panel currents, as described in Section III, is shown in Figure 4. Figure 4a) shows the magnitude of the calibrated PNI data with the IGRF magnitude, and by visual comparison to Figure 2b), we see there is significant improvement. Figure $4 \mathrm{~b}$ ) shows the results of calibration of IMU magnetometer data. From the plot, we see that the IMU calibration did not yield results as close to the IGRF magnitude as the PNI calibration did. The difference between the calibrated IMU magnitude and the IGRF magnitude over the first 500 seconds of data is shown in Figure 5. There is a periodic spike in the error every 20 seconds. This is the frequency of periodic telemetry updates transmitted by RAX (beacons).

In the satellite telemetry, the beacons are seen as peri-

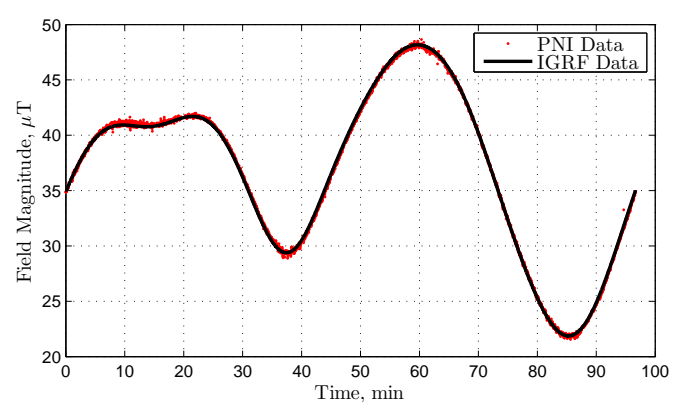

a) PNI and IGRF field magnitude

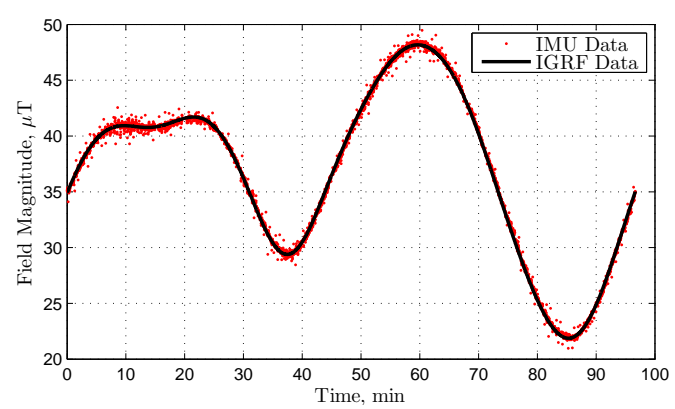

b) IMU and IGRF field magnitude

Figure 4. Results of calibration with the solar panel current measurements. We see significant improvement over the calibration without time-varying parameters (compare Figure 4a) to Figure 2b)).

odic spikes in the current draw from the electrical power system (EPS). To take the magnetic effect of beaconing into account, we include the measurement of current draw from the EPS in the same manner that we included solar panel currents in the magnetometer model. We use the additional telemetry point and estimate three more parameters, which are the mapping of the EPS current draw to magnetometer biases and are analogous to the parameters $s_{i, j}$ for the solar panel currents. The resulting IMU field magnitude and difference between the IMU and IGRF magnitudes are shown in Figure 6. Figure 6a) shows the magnitude of the resulting IMU measurements, and can be compared directly to Figure $4 b$ ). Figure $6 b$ ) shows the difference between the IMU and IGRF magnitudes, and can be compared directly to Figure 5. Both plots show that the periodic error is removed and the measurement accuracy is improved.

\section{A. Calibration Accuracy}

We measure the accuracy of the calibration by the square root of the mean squared error (RMSE), where error is defined as the difference between the IGRF and measured field magnitudes. We have made no assumptions about the 
probability distribution of the data, nor have we attempted to characterize the accuracy of the individual calibration parameters. The statistical analysis is beyond the scope of this paper and is left for future work.

The RMSE of the three calibrations - (1) calibration with no time-varying parameters, (2) calibration with solar panel currents, and (3) calibration with both solar panel currents and EPS current draw - are shown in the bottom row of Tables 1 and 2. The RMSE after calibration with no time-varying parameters was $903 \mathrm{nT}$ and 2,017 nT for the PNI and IMU, respectively. With the calibration that included all five telemetry points, the RMSE was reduced to $174 \mathrm{nT}$ for the PNI and $225 \mathrm{nT}$ for the IMU. The mean of the errors for the final calibration case are $4.1 \mathrm{nT}$ and $0.5 \mathrm{nT}$ for the PNI and IMU, respectively, and histograms of the errors are shown in Figure 7.

The uncertainties of the PNI and IMU magnetometers were measured pre-flight before the magnetometers were integrated into the satellite. This was done by both measuring constant magnetic fields of varying strength generated by a Helmholtz cage [10] and by taking measurements inside a magnetically shielded chamber [12]. The testing showed that the uncertainty is not dependent on magnetic field strength. Defining uncertainty to be the root mean square error, where error is the difference between the measurement and the average measurement of a constant magnetic field, the uncertainty of the magnitude of the measurements is $97 \mathrm{nT}$ for the PNI and $84 \mathrm{nT}$ for the IMU. Although the on-orbit calibration has not reduced the uncertainty quite to the levels of the de-integrated sensors, it is a drastic improvement over the uncertainty of the calibration that does not include time-varying parameters.

\section{B. Summary of Results}

The resulting scaling factors, constant biases, and axis mis-alignments for the three calibrations are given in Tables 1 and 2 . The percent difference between the esti-

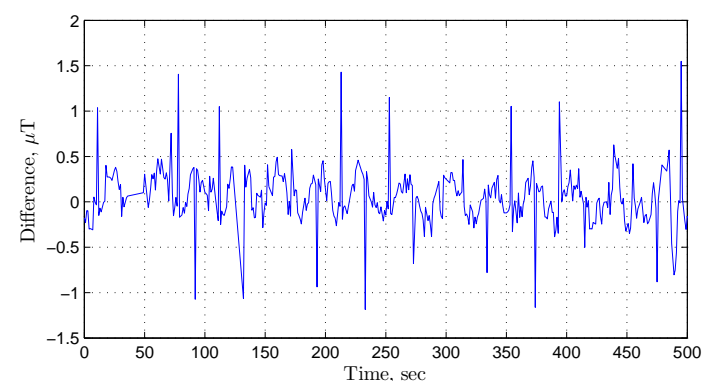

Figure 5. Difference in field magnitude between the calibrated IMU readings and the IGRF data over the first 500 seconds of data shown in Figure 4b). The periodic error is at the same frequency as RAX beacons.

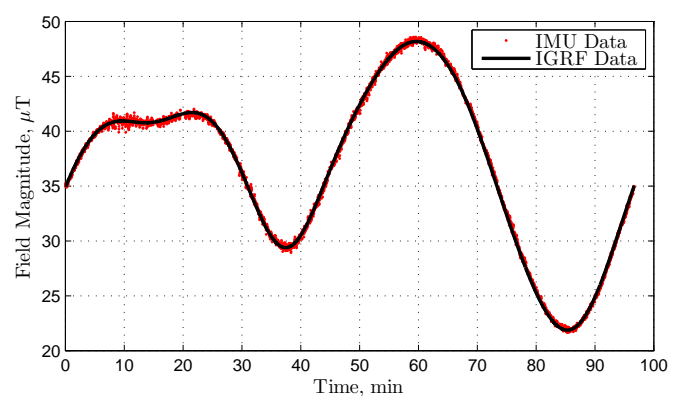

a) IMU and IGRF field magnitude

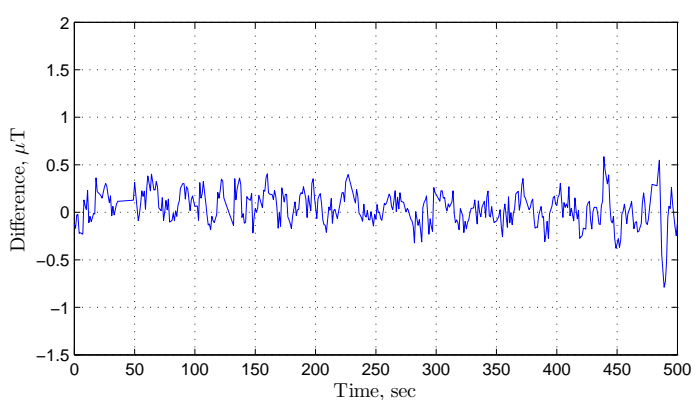

b) Difference in field strength over the first 400 seconds

Figure 6. IMU data after calibration that includes the telemetry of the four solar panels and the current draw from the EPS. This is more accurate than the calibration with only solar panel currents (compare Figure 6a) to 4b), and Figure 6b) to 5).

mated parameters for each calibration are given in the last three columns of the table. There are significant differences between the calibration with no spacecraft telemetry and the calibrations with spacecraft telemetry. This is expected because the calibration without the telemetry attempts to capture time-varying events in constant parameters. The parameters from calibrations 2 and 3 differ by less than $2 \%$. We see that inclusion of current draw from the EPS significantly improves the accuracy of the IMU calibration, but has less of an effect on the PNI calibration. This can be attributed to the physically different locations of the magnetometers in the satellite.

\section{Conclusion}

We have described a method for attitude-independent, on-orbit magnetometer calibration that takes into account time-varying effects of the spacecraft environment. Two specific modifications were made to existing calibration techniques. First, we have implemented a numerical method to estimate calibration parameters under a dynamic magnetic field, and second, we have modified the magnetometer model to include time-varying spacecraft telemetry. Inclusion of the telemetry allows us to esti- 
Table 1. Scale factors, biases, and angular misalignments, and RMSE of the PNI magnetometer calibrations.

\begin{tabular}{c|ccc|ccc}
\hline \hline & \multicolumn{3}{|c|}{ Parameter Estimates } & \multicolumn{3}{c}{ Percent Difference } \\
Parameter & Calib 1 & Calib 2 & Calib 3 & 1 \& 2 & $2 \&$ 3 & 1 \& 3 \\
\hline$a$ & 0.891 & 0.890 & 0.890 & 0.11 & 0.00 & 0.11 \\
$b$ & 0.915 & 0.910 & 0.910 & 0.58 & 0.00 & 0.58 \\
$c$ & 1.133 & 1.130 & 1.130 & 0.33 & 0.00 & 0.33 \\
$x_{0}(\mu \mathrm{T})$ & -0.756 & -0.697 & -0.687 & 7.79 & 1.45 & 9.12 \\
$y_{0}(\mu \mathrm{T})$ & 9.738 & 9.898 & 9.909 & 1.64 & 0.11 & 1.75 \\
$z_{0}(\mu \mathrm{T})$ & -8.212 & -7.679 & -7.701 & 6.49 & 0.28 & 6.23 \\
$\rho\left({ }^{\circ}\right)$ & -1.555 & -1.038 & -1.039 & 33.24 & 0.09 & 33.18 \\
$\phi\left(^{\circ}\right)$ & -4.227 & -3.974 & -3.974 & 5.98 & 0.01 & 5.99 \\
$\lambda\left(^{\circ}\right)$ & 5.443 & 5.018 & 5.019 & 7.81 & 0.01 & 7.80 \\
\hline RMSE (nT) & 903 & 176 & 174 & & & \\
\hline \hline
\end{tabular}

Table 2. Scale factors, biases, and angular misalignments, and RMSE of the IMU magnetometer calibrations.

\begin{tabular}{c|ccc|ccc}
\hline \hline & \multicolumn{3}{|c|}{ Parameter Estimates } & \multicolumn{3}{c}{ Percent Difference } \\
Parameter & Calib 1 & Calib 2 & Calib 3 & $1 \& 2$ & $2 \&$ 3 & $1 \& 3$ \\
\hline$a$ & 0.880 & 0.886 & 0.886 & 0.73 & 0.01 & 0.73 \\
$b$ & 0.905 & 0.903 & 0.903 & 0.22 & 0.01 & 0.22 \\
$c$ & 1.055 & 1.053 & 1.052 & 0.19 & 0.03 & 0.21 \\
$x_{0}(\mu \mathrm{T})$ & -15.070 & -15.104 & -15.106 & 0.23 & 0.01 & 0.24 \\
$y_{0}(\mu \mathrm{T})$ & -4.532 & -3.472 & -3.527 & 23.39 & 1.60 & 22.16 \\
$z_{0}(\mu \mathrm{T})$ & 16.425 & 16.127 & 15.933 & 1.81 & 1.20 & 2.99 \\
$\rho\left(^{\circ}\right)$ & -0.629 & -0.319 & -0.312 & 49.36 & 1.94 & 50.34 \\
$\phi\left(^{\circ}\right)$ & -3.003 & -1.961 & -1.975 & 34.68 & 0.72 & 34.21 \\
$\lambda\left(^{\circ}\right)$ & -0.526 & -1.784 & -1.779 & 239.28 & 0.31 & 238.24 \\
\hline RMSE (nT) & 2,017 & 290 & 225 & & & \\
\hline \hline
\end{tabular}

mate constant parameters that map time-varying current to magnetometer bias, rather than attempting to estimate time-varying parameters. This modeling technique can be extended to include other sources of time-varying bias. The effectiveness of the calibration has been demonstrated by application to on-orbit data from the RAX satellite. We have reduced the RAX magnetometer uncertainty from $903 \mathrm{nT}$ to $174 \mathrm{nT}$ for the PNI, and from 2,017 nT to 225 $\mathrm{nT}$ for the IMU.

This work was motivated by magnetometer-based attitude determination. Angular uncertainty of magnetometers is dependent on the local magnetic field strength. The approximate angular uncertainties after each calibration are shown in Tables 3 and 4 for field strengths of $20 \mu \mathrm{T}$ and $50 \mu \mathrm{T}$, which is the expected range for the RAX orbit. The calibrations have improved the worst-case angular uncertainties of the PNI and IMU magnetometers by $2.1^{\circ}$ and $5.2^{\circ}$, respectively. Although the overall accuracy of an attitude determination system depends on a number of other factors, such as the uncertainty of other attitude sensors and characteristics and sampling frequency of rate gyros, this shows the importance of the magnetometer calibration for attitude determination. The calibration is also applicable to scientific magnetometer measurements.

The calibration relies on the magnitude of the true magnetic field. For space applications, the IGRF is an example source for this truth data. The uncertainty of the IGRF is expected to be approximately $10 \mathrm{nT}$ during normal space weather conditions [11]. In general, the accuracy of the calibration is limited by the accuracy of the truth model.

This work has implications on the satellite design process. Since we have demonstrated the ability to estimate magnetometer non-orthogonality, scaling factors, and time-varying bias on-orbit, satellite designers can put magnetometers anywhere in the spacecraft without loca- 


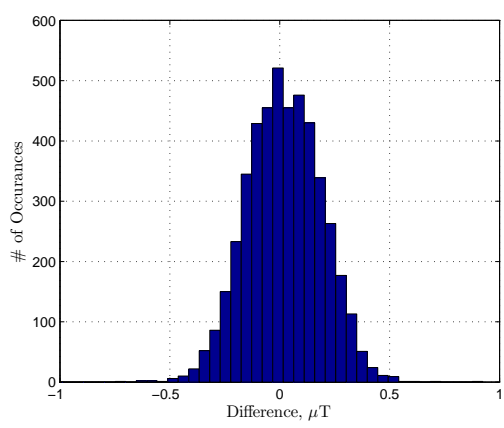

a) PNI Data

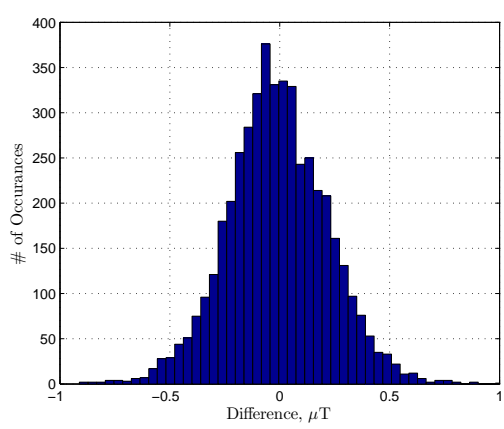

b) IMU Data

Figure 7. Histogram of PNI and IMU magnitude error after calibration with solar panel currents and EPS current draw.

tion constraints due to magnetic cleanliness requirements. Because of this, use of the calibration method reduces both the design time and cost for satellite development.

\section{Acknowledgments}

This work is a subset of magnetometer calibration work done in collaboration with Creare, Inc. and funded by the Air Force Research Laboratory (SBIR contract number FA9453-10-C-0056). RAX is funded by the National Science Foundation (grant ATM-0838054). Additionally, I'd like to thank Sara Spangelo and Ted Beach for their helpful feedback.

\section{References}

[1] Roberto Alonso and Malcolm D. Shuster. Attitudeindependent magnetometer-bias determination: A survey. Journal of the Astronautical Sciences, 50(4):453 - 475, 2003.

[2] Roberto Alonso and Malcolm D. Shuster. Complete linear attitude-independent magnetometer calibration. Journal of the Astronautical Sciences, 50(4):477 - 490, 2003.
Table 3. PNI angular uncertainties corresponding to the RMSEs given in Table 1

\begin{tabular}{c|ccc}
\hline \hline Field Strength & Calib 1 & Calib 3 & Improvement \\
\hline $20 \mu \mathrm{T}$ & $2.6^{\circ}$ & $0.5^{\circ}$ & $2.1^{\circ}$ \\
$50 \mu \mathrm{T}$ & $1.0^{\circ}$ & $0.2^{\circ}$ & $0.8^{\circ}$ \\
\hline \hline
\end{tabular}

Table 4. IMU angular uncertainties corresponding to the RMSEs given in Table 2

\begin{tabular}{c|ccc}
\hline \hline Field Strength & Calib 1 & Calib 3 & Improvement \\
\hline $20 \mu \mathrm{T}$ & $5.8^{\circ}$ & $0.6^{\circ}$ & $5.2^{\circ}$ \\
$50 \mu \mathrm{T}$ & $2.3^{\circ}$ & $0.3^{\circ}$ & $2.0^{\circ}$ \\
\hline \hline
\end{tabular}

[3] Roberto Alonso and Malcolm D. Shuster. TWOSTEP: A fast robust algorithm for attitudeindependent magnetometer-bias determination. Journal of the Astronautical Sciences, 50(4):433 451, 2003.

[4] Bruce T. Blaylock. Spacecraft Attitude Determination and Control, chapter 6.3: Magnetometers, pages 180-184. Kluwer Academic Publishers, 1978.

[5] J.L. Crassidis, Kok-Lam Lai, and R.R. Harman. Real-time attitude-independent three-axis magnetometer calibration. Journal of Guidance, Control, and Dynamics, 28(1):115 - 20, 2005.

[6] John L. Crassidis and John L. Junkins. Optimal Estimation of Dynamical Systems. Chapman and Hall/CRC, 2004. Section 1.4.

[7] James Cutler, Matthew Bennett, Andrew Klesh, Hasan Bahcivan, and Rick Doe. The radio aurora explorer - a bistatic radar mission to measure space weather phenomenon. In Proceedings of the 24th Annual Small Satellite Conference, Logan, Utah, August 2010.

[8] C.C. Foster and G.H. Elkaim. Extension of a twostep calibration methodology to include nonorthogonal sensor axes. IEEE Transactions on Aerospace and Electronic Systems, 44(3):1070 - 8, July 2008.

[9] D. Gebre-Egziabher, G. H. Elkaim, J. D. Powell, and B. W. Parkinson. A nonlinear, two-step estimation algorithm for calibrating solid-state strapdown magnetometers. In Proc. Int. Conf. Integrated Navigation Systems, St. Petersburg, Russia, May 2001.

[10] Andrew Klesh, Sheryl Seagraves, Matt Bennett, Dylan Boone, James Cutler, and Hasan Bahcivan. Dynamically driven helmholtz cage for experimental magnetic attitude determination. In Astrodynamics 2009, volume 135, pages 147-160, 2010. 
[11] S. Maus, S. Macmillan, F. Lowes, and T. Bondar. Evaluation of candidate geomagnetic field models for the 10th generation of IGRF. Earth, Planets, and Space, 57(12):1173-1181, 2005.

[12] John C. Springmann, James W. Cutler, and Hasan Bahcivan. Magnetic sensor calibration and residual dipole characterization for application to nanosatellites. In Proceedings of the AIAA/AAS Astrodynamics Specialist Conference, Toronto, August 2010.

[13] David A. Vallado and Wayne D. McClain. Fundamentals of Astrodynamics and Applications. Springer, 2007. Section 2.4.2.

[14] J. Vasconcelos, G. Elkaim, C. Silvestre, P. Oliveira, and B. Cardeira. A geometric approach to strapdown magnetometer calibration in sensor frame. In Proc IFAC Workshop on Navigation, Guidance, and Control of Underwater Vehicles (NGCUV), Killaloe, Ireland, April 2008. 